\title{
Investigation of the Suitability of IrBurst for High-Speed Exchange of Large Data Blocks
}

\author{
Alam Mohammad Shah, Shawkat Shamim Ara and Mitsuji Matsumoto \\ GITS, Waseda University, Tokyo, Japan
}

\begin{abstract}
This paper provides a mathematical model with which we derive a simple equation for IrBurst throughput efficiency over IrDA protocol stacks. Based on this model, we compare the performance of IrBurst protocol with the existing OBEX protocol for large data blocks exchange at high data rates considering both error free and erroneous environment in order to investigate the suitability of IrBurst protocol. A study of the importance of parameters such as IrLAP window size and IrPHY minimum turnaround time on IrBurst performance is also presented. Finally, a protocol improvement that employs an effective Automatic Repeat Request error control scheme to provide robustness at high bit error rate is proposed.
\end{abstract}

\section{INTRODUCTION}

Traditional mobile devices such as cell phones, PDAs and MP3 players are now required to handle complex jobs involving larger files, such as MP3 audio and MPEG video at high-speed [1]. Because of limited processing power, these devices require a light implementation of IrDA protocols to provide maximum transfer performance. However, this demand for efficient transmission of large data and media contents between mobile devices at increasingly higher speeds calls into question the scalability of the existing IrOBEX [2] protocol designed for object exchange. To compensate the insufficiency of OBEX protocol, IrDA has recently proposed IrBurst, a higher layer protocol, which is particularly designed for high-speed transmission of largescale information [1].

The IrBurst issue has already been examined in [3] but the result presented in the analysis is not sufficient for the details and complete performance analysis of IrBurst. This paper provides a mathematical model with which we derive a simple equation for IrBurst throughput efficiency over IrDA protocol stacks. The model is validated by comparing simulation with analytical results and is employed to compare the performance of IrBurst protocol with existing OBEX protocol for high-speed large data blocks exchange both in error free and erroneous transmission environment as well as to evaluate its performance in details. We also study the importance of physical and link layer parameters on the IrBurst throughput efficiency for high-speed IrDA links $(100 \mathrm{Mb} / \mathrm{s})$ at various bit error rates (BERs). Finally, a protocol improvement that employs an effective Automatic Repeat Request error control scheme to provide robustness at high bit error rate is proposed. A preliminary version of this work appeared in [4].

The rest of the paper is organized as follows: In section II we briefly discuss the limitation of existing OBEX protocol and the overview of IrBurst protocol. In section III we introduce the mathematical model on which we have based our study. The validation of our mathematical model is carried out in Section IV and numerical results are presented in section V. An effective ARQ scheme is proposed in section VI to improve IrBurst protocol performance. Finally section VII presents our conclusions.

\section{PROTOCOL DESCRIPTION}

\section{A. The existing $O B E X$ protocol}

OBEX [2] is a binary session layer protocol of IrDA protocol set designed to transmit data objects between resource-limited devices. It is based on binary HTTP protocol.

OBEX follows a client/server request-response (stop and wait) paradigm for the conversation format $[2,5]$. The "Stop \& Wait" scheme is only fit for small information exchange. However, this scheme is disadvantageous for large-scale information exchange because of the halfduplex nature of IrDA links. OBEX transmits objects using GET and PUT methods. The OBEX 1.3 specification defines a 2 byte packet length header field resulting in a maximum OBEX packet length of $64 \mathrm{~KB}$ [2]. This packet size limitation requires that GET and PUT operations for large objects be segmented into multiple OBEX packets. The OBEX standard requires that each request packet transmitted in either a GET or PUT operation must be acknowledged by a CONTINUE response packet [2]. Fig. 1 shows the OBEX conversation format.

\section{B. IrBurst protocol}

IrBurst is a higher layer protocol designed over TinyTP [6], IrLMP [7], IrLAP [8] and IrPHY [9] for high speed exchange of large-scale information. It uses the burst transmission capability (maximum window size) of the lower layer protocols of IrDA protocol stacks for transmitting large bursts of information [1].

The significant feature of IrBurst protocol is that it adds no extra overhead for the transmission of information body. The extra overhead is avoided due to another convenient capability of the lower layer protocols. The capability is multiple logical channels by Logical Service Access Point (LSAP) [7]. At first, the initiator starts to control high-speed transmission using the control channel. After negotiation, a new data transmission channel is prepared that operates as a stream (see Fig.2). "UPLOAD" 


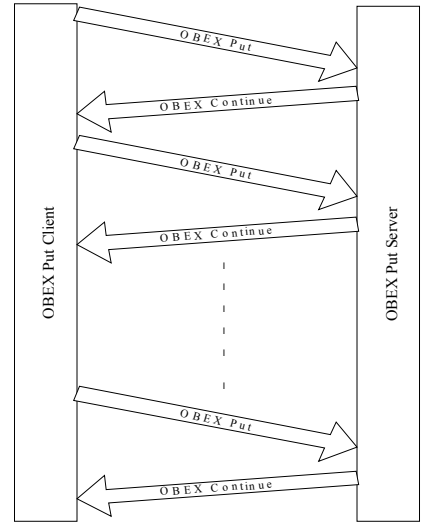

Figure 1. OBEX conversation.

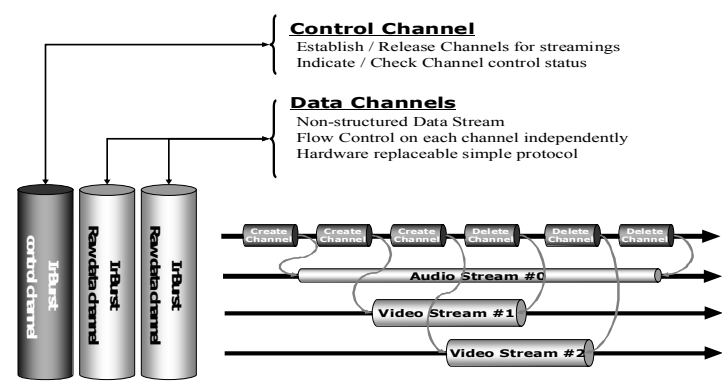

Figure 2. IrBurst overview

and "DOWNLOAD" are the two types of operation used in IrBurst. The information sent is not peer acknowledged at the IrBurst layer unlike the OBEX protocol. The conversation format of IrBurst is shown in Fig.3.

\section{IRBURST AND OBEX MODEL}

In order to calculate IrBurst throughput efficiency over IrDA protocol stacks, a simple equation is derived both in error free transmission and in presence of transmission errors. For simplicity, we derive IrBurst throughput efficiency by considering IrBurst in connected data

TABLE I.

MATHEMATICAL Model VARIABLES

\begin{tabular}{|c|c|c|}
\hline Symb. & Parameter Description & Unit \\
\hline$C$ & Link data rate & $\mathrm{bit} / \mathrm{s}$ \\
\hline$p$ & Frame error probability & \\
\hline$p_{b}$ & Link bit error rate (BER) & \\
\hline$L$ & Data block size & bit \\
\hline$N$ & IrLAP window size & frames \\
\hline$l$ & Payload size of IrLAP frame(Frame length ) & bit \\
\hline$l_{P H Y}$ & Physical layer overhead & $48 \mathrm{bit}$ \\
\hline$l_{L A P}$ & S-frame size/ I-frame (IrLAP) header & $24 \mathrm{bit}$ \\
\hline$l_{L M P}$ & IrLMP layer header & $16 \mathrm{bit}$ \\
\hline$l_{T T P}$ & TinyTP layer header & $8 \mathrm{bit}$ \\
\hline$l_{t}$ & Payload size of TinyTP packet & $\mathrm{bit}$ \\
\hline$t_{t a t}$ & IrLAP minimum turnaround time & $\mathrm{sec}$ \\
\hline$b_{t a t}$ & Equivalent bits of IrLAP turnaround time: & $\mathrm{bit}$ \\
& $C \times$ ttat & \\
\hline$l_{O B E X I}$ & Overhead of first OBEX packet & $\mathrm{bit}$ \\
\hline$l_{O B E X n}$ & Overhead of all subsequent OBEX packets & $48 \mathrm{bit}$ \\
\hline$P_{R E Q}$ & OBEX request packet size & $\mathrm{bit}$ \\
\hline$P_{R E S}$ & OBEX response packet size & $\mathrm{bit}$ \\
\hline$T_{T A}$ & OBEX turnaround time & $\mathrm{sec}$ \\
\hline$b_{T A}$ & Equivalent bits of OBEX turnaround time: & $\mathrm{bit}$ \\
& $C \times T_{T A}$ & \\
\hline
\end{tabular}

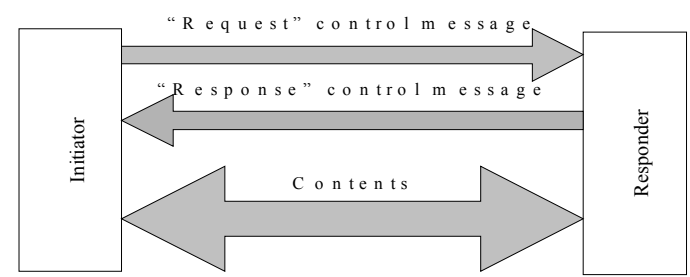

Figure 3. IrBurst conversation

transmission mode and only one application. We also assume that the block is transmitted in the IrBurst "UPLOAD" operation mode. However, the derived model can also be modified in a straightforward manner for "DOWNLOAD" operation mode. Furthermore; we also construct a model for deriving OBEX throughput efficiency for performance comparison. Due to space limitation, both IrBurst model and OBEX model considering only erroneous environment is provided here. Variables presented in this section are defined in Table I.

\section{A. IrBurst Throughput Efficiency in erroneous data transmission}

The protocol mapping of a large data block in IrBurst down to the link layer of the stacks (IrLAP) is illustrated in Fig.4. We consider fixed overheads of 2 bytes and 1 byte for IrLMP and TinyTP respectively. Since all TinyTP packets must fit within a single IrLAP payload, the payload of each TinyTP packet $\left(l_{t}\right)$ is given by

$$
l_{t}=l-l_{T T P}-l_{L M P}
$$

Total TinyTP packets required for data block of size $L$ is:

$$
n=\left\lceil\frac{L}{l_{t}}\right\rceil
$$

Since each packet fits within a single frame and each window has $N$ frames, we divide $n$ by $N$ to yield the total number of windows $(W)$ required to transmit the block without any error.

$$
W=\left\lceil\frac{n}{N}\right\rceil
$$

However, when an error occurs, the erroneous frame and all the subsequent frames in the window needs to be retransmitted which results in increase the number of windows.

Referring to [10], the frame error probability $p$ and the number of frames correctly transmitted in one full window transmission $N_{\text {corr }}$ is given by:

$$
\begin{gathered}
p=1-\left(1-p_{b}\right)^{l+l L A P+l P H Y} \\
N_{\text {corr }}=\frac{(1-p)\left(1-(1-p)^{N}\right)}{p}
\end{gathered}
$$

Therefore, the total number of windows required to transmit the same data content in presence of errors is:

$$
W_{\text {err }}=\frac{L}{\left(l-l_{\text {TTP }}-l_{L M P}\right) * N_{\text {corr }}}
$$

Because IrLAP turnarounds are dependent upon windows, therefore we multiply $W_{\text {err }}$ by $b_{\text {tat }}$ to consider the overhead due to turnaround time in terms of bit.

$$
O_{\text {taterr }}=b_{\text {tat }} *\left\lceil W_{\text {err }}\right\rceil
$$




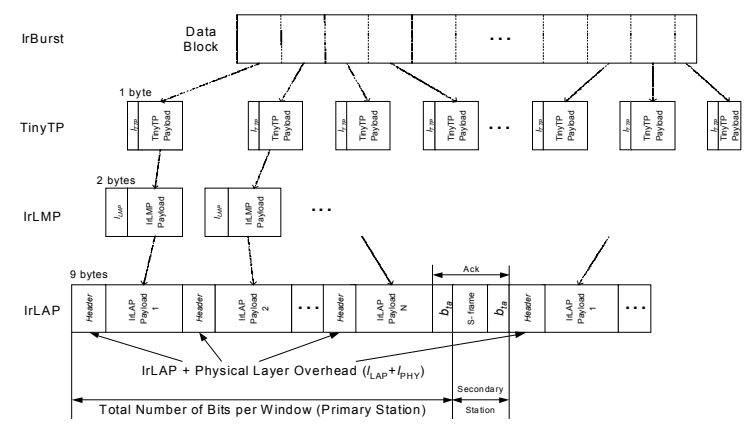

Figure 4. Mapping IrBurst, TinyTP, IrLMP to IrLAP frames

To include the overhead due to lower layer, we add the overhead of each layer and multiply it by the total number of packets to yield total overhead for lower layers.

$$
H=\left(l T T P+l L M P+l_{L A P}+l_{P H Y}\right) * n
$$

Since the erred frame and the subsequent frames of the window are retransmitted in case of error, we subtract total number of windows to transmit the block without any error $W$ from $W_{\text {err }}$ to yield the extra windows which are retransmitted for error and multiply by $N$ to find out the total number of retransmitted frames. Finally we multiply this with frame size $\left(l+l_{L A P}+l_{P H Y}\right)$ to yield the total number of bits which are retransmitted for error:

$$
b R=(l+l L A P+l P H Y) *(N *(\text { Werr }-W))
$$

Furthermore, if the last frame of the window is not correctly received, the $\mathrm{P}$ bit is also lost and the receiver does not respond as it is unaware of link reversion. The primary waits for an F-timer expiration and sends Supervisory frame ( $S$-frame) [8] forcing the receiver to respond. Assuming S-frames are always received correctly, the $\mathrm{P}$ bit loss incorporates an additional frame of size $l_{L A P}+l_{P H Y}$ transmission and the associated turnaround time. Since frame error probability is $p$, we multiply this by $p$ and therefore the total number of bits required to transmit in case of $\mathrm{P}$ bit lost for all windows is:

$$
b_{\text {Plost }}=\left\lceil W_{\text {err }}\right\rceil * p *\left(\left(l_{L A P}+l_{P H Y}\right)+b_{\text {tat }}\right)
$$

As illustrated in the figure, for transmitting the content of size $L$, in addition to this content extra bits are also transmitted which accounts for the overhead of lower layers and turnaround time effect. Moreover, in case of error, the number of bits required to transmit the same

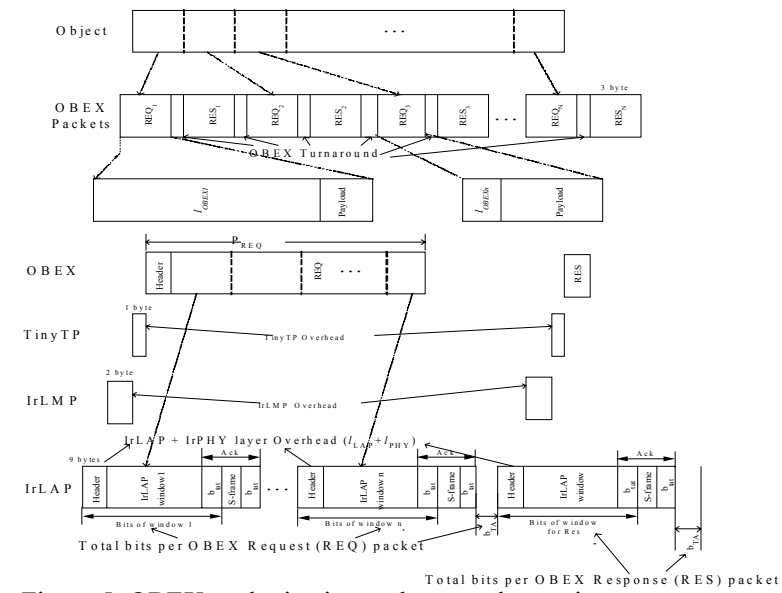

Figure 5. OBEX packetization and protocol mapping content $(L)$ is increased further due to retransmission associated with lost frames and solution of $\mathrm{P}$ bit lost.

Considering all the overheads and effect of errors, we add (7), (8), (9), (10) and data block size $(L)$ to calculate the total number of bits required to transmit the block of size $L$ in presence of transmission errors which is given by:

$$
\text { Berr }=\text { Otaterr }+H+b R+b \text { Plost }+L
$$

The throughput efficiency (TE), which is defined as the ratio of data block size (in bits) to total number of bits required to transmit that block, is therefore in presence of transmission errors is:

$$
T E=\frac{L}{B e r r}
$$

\section{B. OBEX Throughput efficiency in erroroneous data transmission}

In order to derive OBEX throughput efficiency, we assume that the packets are sent in OBEX 'PUT' operation mode and considered only the connected OBEX packets for simplicity. Fig.5 illustrates the way in which OBEX packetizes a large object of same size $(L)$ for transmission and the protocol mapping of this packet down to the link layer of the stacks. The first packet typically contains some extra information $\left(l_{O B E X I}-l_{O B E X n}\right)$ of the object (such as name, length, etc.) but all the subsequent packets contains fixed header of length $l_{O B E X n}$ only.

The total number of OBEX packets for an object of size $L$ is given by:

$$
n 1=\left\lceil\frac{(L+(\text { lOBEX } 1-\text { lOBEXn }))}{\left(\text { PREQ }- \text { lOBEXn }_{n}\right)}\right\rceil
$$

Since TinyTP and IrLMP add their overhead to each OBEX packet when it passes down to the link layer and payload of each IrLAP frame is $l$, therefore the total IrLAP frames required for each OBEX packet is:

$$
n 2=\left\lceil\frac{\left(P_{R E Q}+l_{T T P}+l_{L M P}\right)}{l}\right\rceil
$$

Now using (5), the number of windows required to transmit each OBEX packet in case of error is:

$$
w 1=\left\lceil\frac{n 2}{N_{\text {corr }}}\right\rceil
$$

The OBEX standard requires that each request (REQ) packet must be acknowledged by a response (RES) packet. Because of the half duplex nature of IrDA, subsequent request packets cannot be transmitted until the corresponding response is received. Therefore, to transmit the object in REQ packets, both the overhead associated with request packet and response packet are considered.

Each request/response pair generates two OBEX turnarounds. Considering this higher layer turnaround effect associated with all the REQ packets, we multiply $b_{T A}$ with $n 1$. To this we add the total overhead due to IrLAP turnaround time $\left(n 1^{*} w l^{*} b_{\text {tat }}\right)$ and total overhead for all layers considering all the packets $\left(n 1^{*}\left(n 2^{*}\left(l_{P H Y}+\right.\right.\right.$ $\left.\left.\left.l_{L A P}\right)+l_{L M P}+l_{T T P}+l_{O B E X n}\right)\right)$. We also include the extra information related to the object in the first packet. This yields the total overhead $\left(H_{R E Q}\right)$ associated with all the request (REQ) packets for transmitting the object. 


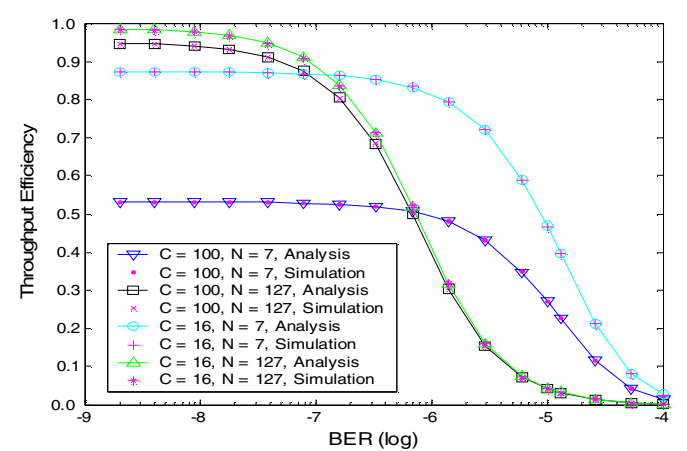

Figure 6. Throughput efficiency: analysis versus simulation

$$
\begin{aligned}
& \text { HREQ }=(n 1 * b T A)+(n 1 * w 1 * b t a t) \\
& +(n 1 *(n 2 *(l P H Y+l L A P)+l L M P+\text { lTTP }+ \text { lOBEXn })) \\
& +(\text { lOBEX } 1-\text { lOBEXn })
\end{aligned}
$$

However, in case of error, the total overhead associated with all request packets for transmitting the same object is increased. This is due to retransmission of erroneous frame and all the subsequent frames in the window and recovery of $\mathrm{P}$ bit lost.

Therefore, the total overhead associated with all Request packets in presence of transmission errors $\left(H_{R E Q e r r}\right)$ is:

$$
\begin{aligned}
& \text { HREQerr }=\text { HREQ } \\
& +\left(\left(\left(\frac{n 2}{N_{\text {corr }}}\right)-\left(\frac{n 2}{N}\right)\right) * N *\left(l+l_{L A P}+l_{P H Y}\right)\right) \\
& +\lceil w 1\rceil * p\left(\left(l_{L A P}+l_{P H Y}\right)+b_{t a t}\right)
\end{aligned}
$$

Similarly, the total overhead $\left(H_{R E S e r r}\right)$ associated with all the response (RES) packets to transmit the object considering no OBEX turnaround time associated with the last response packet is:

$$
\begin{aligned}
& \text { HRESerr }=((n 1-1) * b T A)+(n 1 * b \text { tat }) \\
& +(n 1 *(l P H Y+l L A P+l L M P+l T T P+\text { lOBEXn }))
\end{aligned}
$$

By adding up all these overheads and the size of the object, the total number of bits required to transmit the object of size $L$ is given by:

$$
\text { BOBEX }=\text { HREQerr }+ \text { HRESerr }+L
$$

Therefore OBEX throughput efficiency (TE) in presence of transmission errors is:

$$
T E=\frac{L}{B O B E X}
$$

\section{MODEL VALIDATION}

We have developed a simulation model for IrBurst over IrDA protocol stacks using OPNET ${ }^{\mathrm{TM}}$ simulation package [11]. In our model, all the IrDA protocol details are implemented according to the IrDA specifications. We used point to point connection between primary and secondary station. Bit errors are typically the only source of transmission failure on this point-to-point link. All simulations are run for 1000 seconds of simulated time and the first $10 \%$ of the data is discarded. The performance measurements are logged at the secondary device.

Finally, the simulation results are compared with that obtained from the analytical model in order to validate the model. Fig. 6 plots throughput efficiency versus bit error

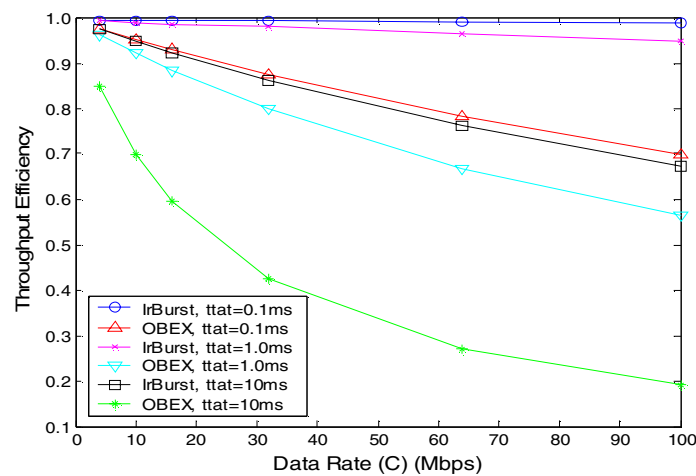

Figure 7. Performance comparison of IrBurst and OBEX for various data rates.

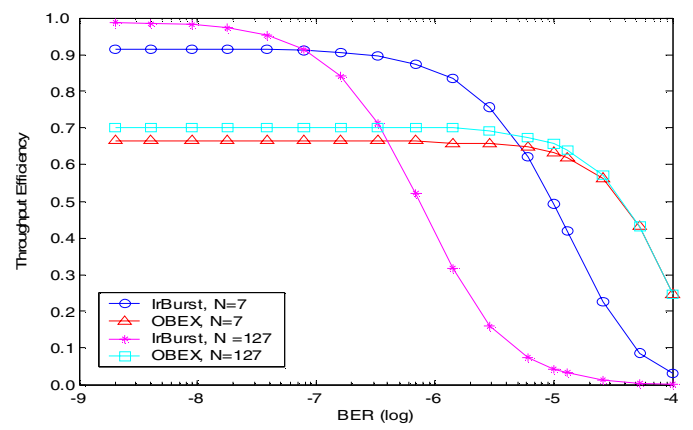

Figure 8. Performance comparison of IrBurst and OBEX for various bit error rates at $C=100 \mathrm{Mb} / \mathrm{s}$

rate (BER) for different window size $(N)$ values with $t_{\text {tat }}=1.0 \mathrm{~ms}, l=2 \mathrm{~KB}$ and $L=100 \mathrm{MB}$ at two different data rates of $100 \mathrm{Mb} / \mathrm{s}$ and $16 \mathrm{Mb} / \mathrm{s}$. The figure shows that analytical results practically coincide with the simulation results for both the data rates. All simulation results in the plot are obtained with a confidence interval of $98 \%$. For space limitation, only the validation of IrBurst model with transmission error (12) is presented here.

\section{NUMERICAL RESUlTS}

\section{A. Performance Comparison of IrBurst and OBEX}

This section provides the performance comparison of IrBurst and OBEX for high-speed exchange of a large data block both in error free and erroneous data transmission based on our model. For this experiment, we used the data content size $(L)$ of $100 \mathrm{MB}$, the IrLAP frame length $(l)$ of $2 \mathrm{~KB}$ and the OBEX turnaround time $\left(T_{T A}\right)$ of $1.0 \mathrm{~ms}$.

Fig.7 compares IrBurst and OBEX throughput efficiency (TE) over a range of data rates with window size of 127 frames and for three different minimum turn around time of $0.1 \mathrm{~ms}, 1 \mathrm{~ms}$ and $10 \mathrm{~ms}$ in case of error free transmission. The figure shows that while a longer minimum turnaround time always degrades throughput efficiency both for IrBurst and OBEX; the effect is more pronounced at higher data transfer rates. At low data rate and very low turnaround time, IrBurst and OBEX have almost the same throughput efficiency. However, as the data rate increases or minimum turn around time increases, the improvement in throughput efficiency (TE) for IrBurst over OBEX also increases. For large value of turnaround time $\left(t_{\text {tat }}=10 \mathrm{~ms}\right)$, IrBurst provides $10 \%$ more TE compared 


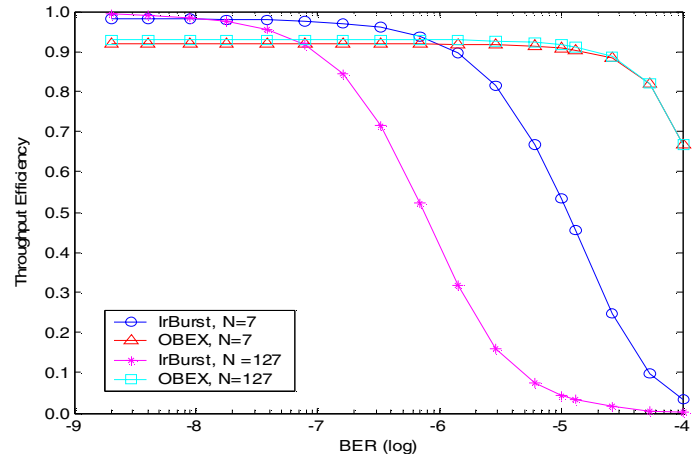

Figure 9. Performance comparison of IrBurst and OBEX for various bit error rates at $C=16 \mathrm{Mb} / \mathrm{s}$.

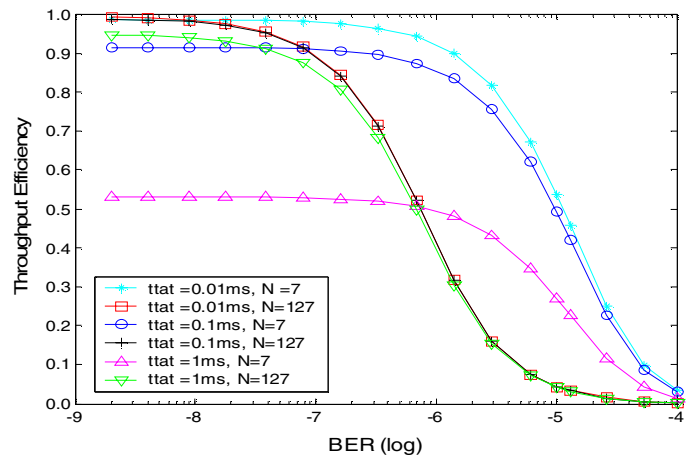

Figure 10. Throughput Efficiency versus BER for $C=100 \mathrm{Mb} / \mathrm{s}$, $L=100 \mathrm{MB}$ and $l=2 \mathrm{~KB}$

to OBEX at $C=4 \mathrm{Mbps}$ while it has $50 \%$ more TE than OBEX at $C=100 \mathrm{Mbps}$. Even for a very low turnaround time of $0.1 \mathrm{~ms}$, IrBurst has almost $30 \%$ improvement in throughput efficiency compared to OBEX at $C=100 \mathrm{Mbps}$. The figure also shows that, IrBurst with low turnaround time $\left(t_{t a t}=0.1 \mathrm{~ms}\right)$ provides excellent throughput efficiency over a range of data rates including high data rate ( $\mathrm{C}=100 \mathrm{Mbps})$.

Using (12) and (20) in Fig.8, IrBurst and OBEX throughput efficiency (TE) are compared over a range of bit error rates (BERs) at $C=100 \mathrm{Mbps}$ with minimum turn around time of $0.1 \mathrm{~ms}$ and two different window sizes of 7 and 127. The figure shows that IrBurst has significant improvement (almost 30\%) in throughput efficiency (TE) over OBEX for window size $(N)$ of 127 at $t_{\text {tat }}=0.1 \mathrm{~ms}$ over a range of bit error rates $\left(10^{-9}\right.$ to $\left.10^{-6}\right)$. For window size ( $N$ ) of 7 , the improvement is nearly $22 \%$ over a wide range of bit error rates $\left(10^{-9}\right.$ to $\left.10^{-5}\right)$. However, as the bit error rate (BER) increases, a much different behavior is observed. At high BER $\left(10^{-6}\right)$, IrBurst performance falls at the same level of OBEX for window size 127 whereas for $N=7$ IrBurst performs better until BER of $10^{-5}$. For further increase in BERs, IrBurst performance decreases significantly while OBEX has better robustness to high BERs.

The same comparison of throughput efficiency (TE) between IrBurst and OBEX considering erroneous data transmission for $16 \mathrm{Mb} / \mathrm{s}$ links is depicted in Fig.9. The figure shows that IrBurst has small improvement in throughput efficiency $(7 \%)$ over OBEX at $C=16 \mathrm{Mb} / \mathrm{s}$ links with a low turnaround time of $0.1 \mathrm{~ms}$ for both

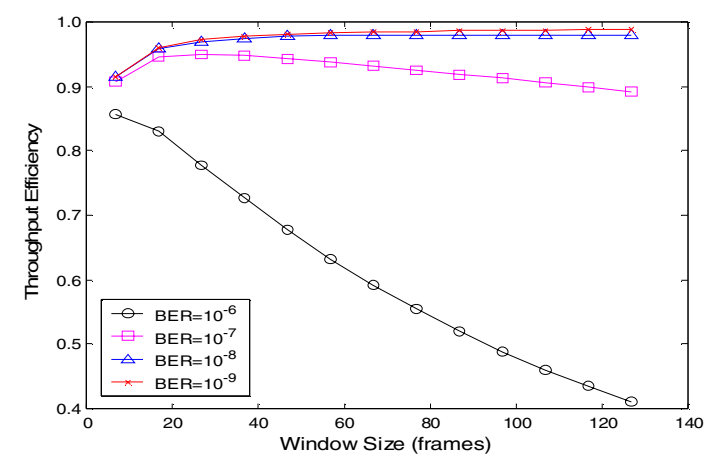

Figure 11. Throughput efficiency versus window size for various bit error rates at $\mathrm{C}=100 \mathrm{Mbps}, t_{\text {tat }}=0.1 \mathrm{~ms}$ and $l=2 \mathrm{~KB}$

window size 7 and 127. This figure confirms that OBEX has significant robustness to high BERs for $16 \mathrm{Mb} / \mathrm{s}$ links also.

Therefore, IrBurst protocol significantly outperforms existing OBEX protocol for exchange of large data blocks especially at high data rates and its throughput efficiency always benefits by a large window size $(N=127)$. However, the OBEX protocol provides high robustness to the BER increase compared to the IrBurst protocol. This is due to the use of Stop and Wait error control scheme at OBEX layer in addition to the lower layer error recovery scheme.

\section{B. IrBurst Performance Evaluation in Presence of Transmission Errors}

In order to carry out details performance evaluation of IrBurst in presence of transmission errors, we examine our model (12) with a variation of different parameters. Fig. 10 plots throughput efficiency versus BER for $C=100 \mathrm{Mb} / \mathrm{s}, l=2 \mathrm{~KB}, L=100 \mathrm{MB}$ and different values of $t_{\text {tat }}$ and $N$. The figure shows that for very low turnaround time of $0.01 \mathrm{~ms}$ or less, window size 7 with existing frame length $(l=2 \mathrm{~KB})$ is sufficient to achieve satisfactory performance for IrBurst over a wide range of BERs. As $t_{\text {tat }}$ increases, IrBurst shows significant improvement in TE for larger window size $(N=127)$ at low BERs but suffers considerable deterioration in TE at high BERs.

However, low turnaround time is not always achievable due to physical limitations and backward compatibility with other device. Therefore, in situations where small turnaround time is not possible, using a large window size $(N=127)$ can alleviate the negative effect of a large minimum turnaround time by increasing the amount of data sent between turnarounds.

Fig. 11 examines the effect of window size on IrBurst throughput efficiency (TE) for different link BERs with $C=100 \mathrm{Mb} / \mathrm{s}, L=100 \mathrm{MB}, \quad l=2 \mathrm{~KB}$ and $t_{\text {tat }}=0.1 \mathrm{~ms}$. The figure depicts that large window size provides significant throughput increase for low BERs but renders the TE very much vulnerable to BER increase. Thus, for large window size, significant decrease in IrBurst TE is observed for high BERs caused by the retransmission of correctly received out of sequence frames. This is a limitation of the existing Go-Back- $N$ (GBN) ARQ scheme adopted by IrDA. Therefore, an effective ARQ scheme is of great importance for IrBurst protocol throughput as well as high-speed IrDA links throughput, especially at high BERs. 


\begin{tabular}{|c|c|c|c|c|c|}
\hline \multicolumn{7}{|c|}{ Existing frame structure } & Extended \\
\hline $\begin{array}{c}\mathrm{Nr} \\
(7 \mathrm{bits})\end{array}$ & $\begin{array}{c}\mathrm{P} / \mathrm{F} \\
(1 \mathrm{bit})\end{array}$ & $\begin{array}{c}\text { Unused } \\
(4 \mathrm{bit})\end{array}$ & $\begin{array}{c}\text { SS } \\
(2 \text { bits })\end{array}$ & $\begin{array}{c}\text { Frame Type } \\
(2 \text { bits })\end{array}$ & Block Status \\
\hline
\end{tabular}

Figure 12 . Supervisory frame structure for proposed ARQ scheme

\section{IMPROVING IRBURST PERFORMANCE}

To improve IrBurst protocol performance, we propose a more effective ARQ scheme at IrLAP layer instead of adaptation of parameters to optimum values. In our proposed ARQ scheme, all error free but out of sequence frames received at the secondary station (receiver) are stored in corresponding positions of a buffer. All frame positions within the receiver buffer are grouped into a number of blocks that is negotiated during the connection establishment phase and the blocks form the units of acknowledgement and retransmissions. If a block consists of $2^{x}$ frames, then the sequence number of a block contains $s-x$ bits, where $s$ is the number of bits in the sequence number of a frame. The $i$ th block contain all frames with sequence numbers in the range $i^{*} t$ to $(i+1)^{*} t$ -1 where $t$ is the number of frames in a block. The secondary station always sends a supervisory frame after getting the poll bit in the final information frame. The modified supervisory frame (Fig. 12) includes the next expected frame number $(\mathrm{Nr})$ and a bit map indicating the status of the blocks in its buffer. Only when a block within the next window has all frames stored in the buffer of secondary station, the corresponding bit position is assigned as ' 1 '. Based on this information, the primary retransmits or transmits only the expected blocks within the next window. The operation of our proposed ARQ scheme is briefly explained in Fig. 13.

Fig. 14 plots throughput efficiency versus bit error rate (BER) for the proposed ARQ scheme and the existing Go-Back-N (GBN) ARQ scheme for $100 \mathrm{Mb} / \mathrm{s}$ link data rate $(C)$ with turn-around time $\left(t_{\text {tat }}\right) 0.1 \mathrm{~ms}$ and IrLAP frame length $(l) 2 \mathrm{~KB}$. It shows that the proposed ARQ scheme enables IrBurst to achieve almost 98\% throughput efficiency (TE) at low BERs by using large window size $(N=127)$ while it has better robustness than GBN scheme with small window size $(N=7)$ at high BERs.

\section{CONCLUSION}

In this article, we carried out a mathematical model to derive a simple equation for IrBurst throughput efficiency (TE) over the IrDA protocol stacks. Based on this model, the performance of IrBurst protocol is compared with existing OBEX protocol both in error free transmission and in presence of transmission errors. We have shown that the IrBurst protocol scales well to handle large data blocks and high data rates compared to the existing OBEX protocol. However, OBEX protocol provides more robustness to the BER increase compared to the IrBurst protocol. Furthermore, the effect of lower layer parameters such as IrLAP window size and IrPHY minimum turnaround time on IrBurst throughput is explored for various BERs. Although very small

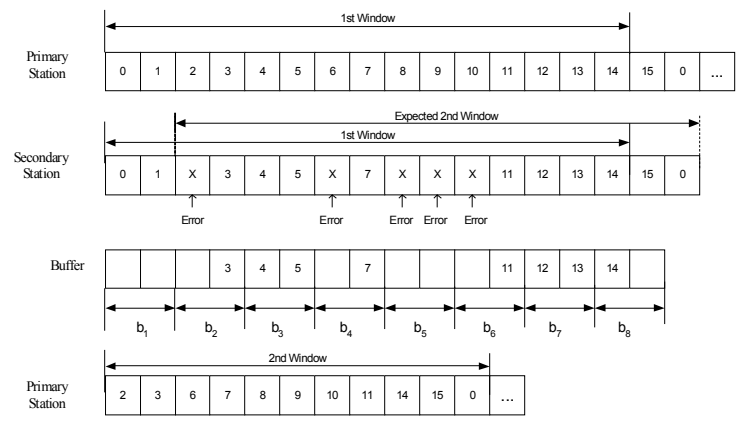

Figure 13. Proposed ARQ scheme for block size of 2 frames and $N=15$ frames

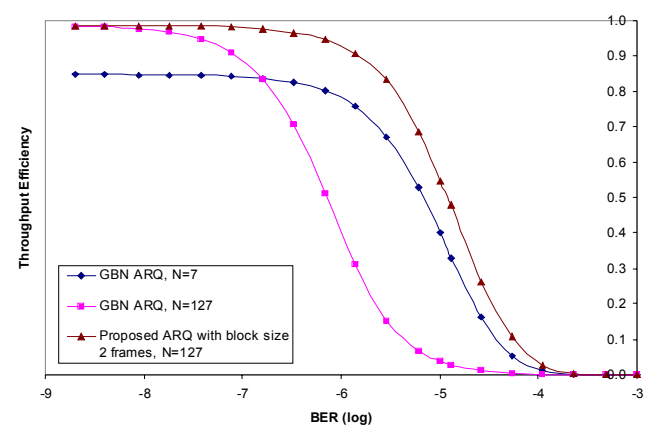

Figure 14. Throughput efficiency versus BER for $t_{t a t}=0.1 \mathrm{~ms}$, $l=2 \mathrm{~KB}, N=127$ and $C=100 \mathrm{Mbit} / \mathrm{s}$

minimum turnaround time results in maximum throughput, such a low turnaround time is not always achievable. Therefore, in situations where small turnaround time is not possible, the use of large window size can mitigate the negative effect of large turnaround time. Finally, an effective ARQ scheme at IrLAP layer is proposed to improve IrBurst throughput performance at high BER when large window size is used. The system performance shows significant improvement by applying the proposed ARQ scheme.

\section{REFERENCES}

[1] IrBurst High-speed Information Transmission Profile, Version 0.9, February, 2005.

[2] IrDA, Object Exchange Protocol (IrOBEX), Version 1.3, March, 2003.

[3] Pi Huang and A. C. Boucouvalas, "Analysis of the High Speed Infrared Information Transmission Protocol: IrBurst," in proceedings of Convergence of Telecommunications, Networking and Broadcasting Symposium 2004 (PGNET 2004), Liverpool, June 2004, pp.328-332.

[4] Shawkat Shamim Ara, Alam Mohammad Shah and Mitsuji Matsumoto, "A Performance Comparison of IrBurst and IrOBEX for High-Speed Exchange of Large Data Contents", accepted for publication in proceedings of IEEE Sarnoff Symposium 2006, Princeton, March 2006

[5] Pi Huang and A. C. Boucouvalas, "OBEX Performance Evaluation and Parameter Optimization for High Speed IrDA Links," IEEE International Conference on Communications (ICC 2004), Paris, June 2004, vol. 7, pp. 3849-3853.

[6] IrDA, Tiny Transport Protocol (IrTinyTP), Version 1.1, 1996.

[7] IrDA, Link Management Protocol (IrLMP), Version 1.1, 1996.

[8] IrDA, Serial Infrared Link Access Protocol (IrLAP), Version 1.1, 1996.

[9] IrDA, Serial Infrared Physical Layer Link Specification, IrPHY, for $16 \mathrm{Mb} / \mathrm{s}$ Addition (VFIR) - Errata to Version 1.3, 1999.

[10] V. Vitsas and A. C. Boucouvalas, "Optimization of IrDA IrLAP Link Access Protocol," IEEE Trans. Wireless Commun., vol.2, no.5, pp 926-938, Sep. 2003

[11] OPNET $^{\mathrm{TM}}$ modeller, MIL3 Inc., 3400 International Drive NW, Washington DC 20008, USA. 